\title{
Scheduling Ambulance Crews for Maximum Coverage
}

\author{
Güneş Erdoğan * $\quad$ Erhan Erkut ${ }^{\dagger} \quad$ Armann Ingolfsson ${ }^{\ddagger}$ \\ Gilbert Laporte*
}

April 14, 2008

\begin{abstract}
This paper addresses the problem of scheduling ambulance crews in order to maximize the coverage throughout a planning horizon. The problem includes the subproblem of locating ambulances to maximize expected coverage with probabilistic response times, for which a tabu search algorithm is developed. The proposed tabu search algorithm is empirically shown to outperform previous approaches for this subproblem. Two integer programming models which use the output of the tabu search algorithm are constructed for the main problem. Computational experiments with real data are conducted. A comparison of the results of the models is presented.
\end{abstract}

Keywords: ambulance location, shift scheduling, tabu search, integer programming.

\footnotetext{
*Canada Research Chair in Distribution Management, HEC Montréal, 3000 Chemin de la CôteSainte-Catherine, Montréal, Canada H3T 2A7 egunes@crt.umontreal.ca, gilbert@crt.umontreal.ca

†Ozyegin University, Kuşbakışı Sokak No:2, Altunizade, İstanbul, Turkey erhan.erkut@ozyegin.edu.tr

${ }^{\ddagger}$ School of Business, University of Alberta, Edmonton, Alberta, Canada Armann.Ingolfsson@ualberta.ca
} 


\section{Introduction}

Effectiveness of Emergency Medical Services (EMS) is a crucial ingredient of an efficient healthcare system. The quality of service for EMS systems is measured according to multiple criteria, including average response time, the type of care that EMS staff are trained to provide, and the equipment to which they have access. The most commonly used indicator of quality of service is the fraction of calls whose response time is within a time standard, typically eight to ten minutes. In planning models, this quantity is often approximated using the concept of coverage, where a demand node is assumed to be covered by an ambulance station if the average response time is within a preset limit. Many studies exist on improving the quality of service of EMS systems. We refer the reader to Goldberg (2004) for a recent review. Although the basic assumptions of such studies vary, based on the perspective of the modeler and the EMS system at hand, one common assumption is the static availability of the service resources. In other words, once an ambulance is introduced into the system, it is assumed to be active at any given time. However, this is usually not the case in real world applications because of the human element involved. As prescribed by laws and legislations governing the working conditions of EMS staff, there are limits on the amount of time and the periods of time an ambulance crew can work in a day. Consequently, there usually exists a limited number of working hour patterns called shifts resulting in time-varying service resources. Scheduling the working hours of EMS staff based on these shifts to maximize coverage is a problem that arises periodically. Even when the scheduling decisions are made, the subproblem of locating ambulances based on the varying number of calls still remains unsolved. When implementing results based on existing ambulance location methods, it is crucial to account for the dynamic availability of resources over time.

The purpose of this paper is to develop a solution method for the combined problem of scheduling the working hours of ambulance crews for a given planning horizon and allocating the ambulances at stations distributed throughout a geographical region. The objective is to maximize expected coverage, taking into account the probabilistic nature of the problem. The core decision is to allocate ambulance crews to shifts, subject to the maximum number of work hours that can be afforded by the decision makers. The output of the crew-shift assignment is the number of ambulances available for every time interval within the planning horizon. The number of ambulances available at a given time interval should respect a lower limit based

on the average number of calls arriving within that time interval. Locating these ambulances to stations in order to maximize expected coverage for the time interval 
while not exceeding the capacity limits of the stations is also a part of the problem.

The complexity resulting from the time element of the problem can be handled by discretizing it, i.e., by dividing the planning horizon into equal-length time intervals. In most applications a planning horizon of one week is appropriate for two reasons: 1) the number of emergency calls received behaves in a cyclic manner with a oneweek period; 2) shifts are usually planned so that staffing is constant from week to week. The length of the time intervals is typically considered to be one hour. A solution method for the shift scheduling problem should be able to assess the result of allocating a given number of ambulances for a given hour of the week in the form of expected number of calls covered within the hour. However, this assessment is an ambulance location problem on its own. Combining a weekly shift scheduling problem and an ambulance location problem for every hour into a single model is likely to result in an intractable model. A useful observation is that once the scheduling decisions are made, the ambulance location problems for each hour become independent of each other. This assumes that ambulances can be moved between stations every hour to achieve an optimal configuration. Based on our observations of real systems, we believe this is a reasonable assumption.

In order to cope with the complexity of the shift scheduling problem, we propose to solve the ambulance location problem for the combinations of the call density of every hour in a week and every possible number of ambulances, and use the results as an input to the shift scheduling problem. To this end, we develop a tabu search algorithm for the hourly ambulance location problem. We then construct two alternative models for the shift scheduling problem, which use the output of the tabu search algorithm and vary by their objective function. The first model aims at maximizing the aggregate expected coverage, i.e., the ratio of the sum of the expected number of calls covered to the total number of calls. The second model is a lexicographic biobjective model, in which the first objective is to maximize the minimum expected coverage over every hour, and the second objective is to maximize the aggregate expected coverage.

The remainder of the paper is organized as follows. In Section 2, we review the existing models for the ambulance location and shift scheduling problems. In Section 3, we develop a tabu search algorithm to solve the subproblem of allocating ambulances to stations and compare our results with those of the previous studies. We construct two integer programming models for the main problem in Section 4. Computational results for both models are presented in Section 5 and conclusions follow in Section 6. 


\section{Review of related literature}

In this section we review the existing literature on the ambulance location models and the shift scheduling models.

\subsection{Ambulance location models}

Ambulance location problems have received a great deal of interest. We refer the reader to Swersev (1994), Marianov and ReVelle (1995), Brotcorne et al. (2003), and Jia et al. (2007) for detailed reviews of the related literature. The paper by Brotcorne et al. (2003) identifies 18 different models for ambulance location. The level of sophistication of a model can be evaluated on its ability to handle the probabilistic nature of the problem, i.e., how expected coverage is computed. The models involving expected coverage follow two tracks:

1) Incorporating the probability that a station may have no ambulances to respond to a call: If the probability of having an idle EMS vehicle at a given station is $p$, then the expected coverage for a demand point within the coverage time limit is not 1 but $p$ (e.g., Daskin 1983, Saydam and McKnew 1985, ReVelle and Hogan 1989).

2) Incorporating response time uncertainty: If the probability of responding from the closest station to a demand point within the given time limit is $q$ and if the closest station has an ambulance, then the expected coverage for that demand point is $q$ (Daskin 1987).

In a model that incorporates both EMS vehicle availability and response time uncertainty, the expected coverage for a unit demand would be $p q$, assuming the two sources of uncertainty are independent. Goldberg and Paz (1991) were the first, to our knowledge, to formulate a mathematical program that addressed both sources of uncertainty. They allowed ambulance busy probabilities to vary between stations and used pairwise exchange heuristics to optimize expected coverage, as evaluated by the Approximate Hypercube (AH) model of Larson (1975). Ingolfsson et al. (2008) made the same assumptions but used a different solution heuristic, one that iterates between solving a nonlinear integer program and the AH model. We will refer to

the problem studied by Goldberg and Paz (1991) and Ingolfsson et al. (2008) as the Maximum Expected Coverage Location Problem with Probabilistic Response Times and Station Specific Busy Probabilities (MEXCLP+PR+SSBP). 


\section{Parameters:}

$n \quad:$ Number of stations.

$m$ : Number of demand nodes.

$q \quad:$ Number of ambulances.

$d_{i} \quad$ : The average number of calls originating at demand node $i$.

$c_{j}$ : The maximum number of ambulances that can be located at station $j$.

$p_{j} \quad$ : The probability that an ambulance located at station $j$ is busy.

$P_{i j}$ : The probability that an ambulance dispatched from station $j$ covers demand node $i$.

$i(j):$ The $j^{\text {th }}$ preferred station for demand node $i$. The preference order is based on the distance between the station and the demand node, with ties broken randomly.

Letting $z_{j}$ be the number of ambulances located at station $j$, the problem can be defined as:

$$
\text { maximize } s\left(z_{1}, \ldots, z_{n}\right)
$$

subject to

$$
\begin{aligned}
& \sum_{j=1}^{n} z_{j} \leq q \\
& z_{j} \in\left\{0,1, \ldots, c_{j}\right\} .
\end{aligned}
$$

where the objective function $s\left(z_{1}, \ldots, z_{n}\right)$ in (1) is the expected number of calls covered, constraint (2) sets the total number of ambulances to be allocated, and constraints (3) set upper bounds on the number of ambulances allocated to each station.

The function $s($.$) has no known closed-form expression and is only defined for$ non-negative integer values of its arguments. It can be evaluated using the $\mathrm{AH}$ model. Alternatively, if one assumes that the status (busy or idle) of one ambulance is independent of the status of all other ambulances (an assumption made, for example, in Daskin (1983) and Goldberg and Paz (1991)), then (11) can be expressed as 


$$
\operatorname{maximize} \sum_{i=1}^{m} d_{i} \sum_{j=1}^{n} P_{i, i(j)}\left(1-p^{z_{i(j)}}\right) \prod_{u=1}^{j-1} p_{i(u)}^{z_{i(u)}}
$$

For a recent study comparing the performance of several ambulance location models including MEXCLP+PR+SSBP, we refer the reader to Erkut et al. (2008).

\subsection{Shift scheduling models}

As underlined in the survey by Goldberg (2004), shift scheduling for ambulances has received almost no attention in the research literature. We refer the reader to Ernst et al. (2004) for a general review on staff scheduling and rostering. Typically, such models decouple performance evaluation from scheduling, by assuming the availability of a set of staffing requirements for each period that if met will guarantee that the quality of service is sufficient. Two notable exceptions are Thompson (1997) and Koole and van der Sluis (2003), both of whom maximize an aggregate quality

of service measure based on an $M / M / s$ queueing model. In contrast, the quality of service measure that we maximize is based on the hypercube queueing model, where the "servers" are spatially distributed and closed-form expressions are not available.

Like most of the shift scheduling literature, we use a steady state approximation to evaluate performance in each period. Green et al. (2001) have investigated such approximations when the system can be modeled as an $M / M / s$ queue and found that although they are often adequate, they are unreliable in certain situations, such as when average service times are relatively long. Ambulances typically take about an hour to handle a call, suggesting that it may be worthwhile to investigate models that incorporate transient effects, but we leave this for future research.

\section{Static allocation of ambulances to stations}

In this section we present a tabu search algorithm to solve the MEXCLP+PR+SSBP. We use a version of the AH model from Budge et al. (2008) that allows for the possibility of multiple ambulances per station to directly compute the expected coverage $s($.$) for a given solution, instead of using the approximation in (44). The$ solution is encoded in a vector $z_{i}$ as the the model given in the previous section. At every iteration, we consider moving a single ambulance from one station to another. 


\section{Parameters:}

$\kappa:$ Number of iterations since the last update of the best solution value.

$\eta$ : The maximum number of iterations without updating the best solution.

$\theta$ : Number of iterations for which a vertex stays in the tabu list.

$\zeta$ : The maximum number of ambulances, i.e., $\zeta=\sum_{i=1}^{n} c_{j}$.

Step 1 (Initialization). Construct a vector $\left(a_{1}, \ldots, a_{\zeta}\right)$ with binary components, corresponding to an actual physical capacity for ambulance storage. The storage space of a station $i$ is represented by entries in the range $\left[s_{i}, t_{i}\right]$, where $s_{1}=1, s_{i}=$ $\sum_{j=1}^{i-1} c_{j}+1$ for $i>1$, and $t_{i}=\sum_{j=1}^{i} c_{j}$. Set the first $q$ components of the vector to be equal to 1 , i.e., $a_{j}=1, \forall j \in\{1, \ldots, q\}$. Set the rest of the components to be equal to zero 0 , i.e., $a_{j}=0, \forall j \in\{q+1, \ldots, \zeta\}$. For $j=1, \ldots, \zeta-1$, swap the value of the $j^{\text {th }}$ component with the value of the $k^{\text {th }}$ component, where $k$ is a randomly selected integer from the interval $[j, \zeta]$. Determine the number of ambulances allocated at station $i$ as $z_{i}=\sum_{j=s_{i}}^{t_{i}} a_{j}$. Evaluate the solution, and record it as the best solution found. Set $\kappa=1$.

Step 2 (Termination check). If $\kappa=\eta$, stop.

Step 3 (Local search). For every location $i$ with $z_{i}>0$ and every location $j \neq i$ with $z_{j}<c_{j}$, evaluate the allocation resulting after moving an ambulance from $i$ to $j$. If the best new allocation has a higher expected coverage value than the best solution found, set the current solution to be the new solution, update the best solution found, and set $\kappa=1$. Else, find the best new allocation for which the station $i$ is not in the tabu list and set the current solution to be the new solution. Add the station that received an ambulance to the tabu list.

Step 4 (Tabu list update). Increase the tabu tenure of each vertex in the tabu list by one. Remove from the tabu list the vertices having a tabu tenure greater than or equal to $\theta$. Increment $\kappa$ by 1 . Go to Step 2.

We have implemented our tabu search algorithm using $\mathrm{C}++$ on a Linux workstation with a 64-bit AMS Opteron 275 CPU running at 2.4GHz. We have conducted computational experiments to compare the performance of our algorithm with the algorithm of Ingolfsson et al. (2008), using real-world data available from http://www.bus.ualberta.ca/aingolfsson/data/. The data consists of the average response times and demand intensity for 16 stations and 180 demand nodes from the city of Edmonton, Canada. Our computational experiments involve two dimensions following the example of Erkut et al. (2008). The first one is the number of 
ambulances, and the second is the system-wide busy-probability. The demand data is scaled based on the system-wide busy-probability to reflect the corresponding call intensity. For the tabu search algorithm, we have used $\eta=20$ and $\theta=\lfloor n / 2\rfloor$. We have performed five replications for each experimental design setting, to eliminate the effect of the random initial solution. Notably, the results of the five replications were always the same for all 126 experimental settings except for one. The performance of our algorithm is never worse than that of the algorithm by Ingolfsson et al. (2008), and is strictly better for 86 out of 126 cases. The average improvement is $0.82 \%$, with the effect becoming more pronounced for higher values of system-wide busy probability. The average CPU time is 50.07 seconds for our tabu search algorithm, as compared to 106.56 seconds of the algorithm by Ingolfsson et al. (2008). Table 1 shows the percent improvement in expected coverage when the tabu search algorithm is used instead of the algorithm by Ingolfsson et al. (2008).

\begin{tabular}{|c|c|c|c|c|c|c|}
\hline & \multicolumn{6}{|c|}{ System-Wide Busy Probability } \\
\hline $\mathbf{q}$ & $\mathbf{0 . 1}$ & $\mathbf{0 . 2}$ & $\mathbf{0 . 3}$ & $\mathbf{0 . 4}$ & $\mathbf{0 . 5}$ & $\mathbf{0 . 6}$ \\
\hline $\mathbf{5}$ & $0.00 \%$ & $0.21 \%$ & $1.40 \%$ & $2.10 \%$ & $3.47 \%$ & $0.00 \%$ \\
$\mathbf{6}$ & $0.00 \%$ & $0.03 \%$ & $0.90 \%$ & $1.62 \%$ & $1.34 \%$ & $1.68 \%$ \\
$\mathbf{7}$ & $0.00 \%$ & $1.60 \%$ & $0.21 \%$ & $2.01 \%$ & $1.06 \%$ & $1.30 \%$ \\
$\mathbf{8}$ & $0.02 \%$ & $0.25 \%$ & $2.96 \%$ & $0.14 \%$ & $0.42 \%$ & $1.18 \%$ \\
$\mathbf{9}$ & $0.37 \%$ & $0.06 \%$ & $1.17 \%$ & $2.51 \%$ & $1.01 \%$ & $1.27 \%$ \\
$\mathbf{1 0}$ & $0.00 \%$ & $0.58 \%$ & $3.03 \%$ & $0.25 \%$ & $3.45 \%$ & $0.93 \%$ \\
$\mathbf{1 1}$ & $0.00 \%$ & $0.20 \%$ & $0.82 \%$ & $0.63 \%$ & $0.96 \%$ & $0.88 \%$ \\
$\mathbf{1 2}$ & $0.00 \%$ & $0.08 \%$ & $0.75 \%$ & $0.00 \%$ & $0.89 \%$ & $2.55 \%$ \\
$\mathbf{1 3}$ & $0.36 \%$ & $0.15 \%$ & $0.26 \%$ & $0.98 \%$ & $0.89 \%$ & $2.06 \%$ \\
$\mathbf{1 4}$ & $0.00 \%$ & $0.26 \%$ & $0.15 \%$ & $0.38 \%$ & $1.71 \%$ & $2.77 \%$ \\
$\mathbf{1 5}$ & $0.00 \%$ & $0.34 \%$ & $0.00 \%$ & $0.00 \%$ & $1.06 \%$ & $2.21 \%$ \\
$\mathbf{1 6}$ & $0.00 \%$ & $0.41 \%$ & $0.35 \%$ & $1.08 \%$ & $0.77 \%$ & $1.26 \%$ \\
$\mathbf{1 7}$ & $0.00 \%$ & $0.40 \%$ & $0.00 \%$ & $0.37 \%$ & $2.04 \%$ & $2.43 \%$ \\
$\mathbf{1 8}$ & $0.00 \%$ & $0.00 \%$ & $0.03 \%$ & $1.95 \%$ & $1.20 \%$ & $1.24 \%$ \\
$\mathbf{1 9}$ & $0.00 \%$ & $0.00 \%$ & $0.00 \%$ & $1.18 \%$ & $1.27 \%$ & $2.75 \%$ \\
$\mathbf{2 0}$ & $0.00 \%$ & $0.00 \%$ & $0.00 \%$ & $0.73 \%$ & $3.08 \%$ & $2.05 \%$ \\
$\mathbf{2 1}$ & $0.00 \%$ & $0.00 \%$ & $0.00 \%$ & $0.22 \%$ & $2.10 \%$ & $2.03 \%$ \\
$\mathbf{2 2}$ & $0.00 \%$ & $0.00 \%$ & $0.00 \%$ & $0.00 \%$ & $2.06 \%$ & $3.31 \%$ \\
$\mathbf{2 3}$ & $0.00 \%$ & $0.00 \%$ & $0.00 \%$ & $0.00 \%$ & $1.34 \%$ & $2.37 \%$ \\
$\mathbf{2 4}$ & $0.00 \%$ & $0.00 \%$ & $0.00 \%$ & $0.07 \%$ & $0.66 \%$ & $2.06 \%$ \\
$\mathbf{2 5}$ & $0.00 \%$ & $0.00 \%$ & $0.00 \%$ & $0.20 \%$ & $0.36 \%$ & $1.73 \%$ \\
\hline Average: & $0.04 \%$ & $0.22 \%$ & $0.57 \%$ & $0.78 \%$ & $1.48 \%$ & $1.81 \%$ \\
\hline
\end{tabular}

Table 1: Percent improvement of expected coverage for the tabu search algorithm.

We have extended our experimentation to the rest of the possible number of 
ambulances and analyzed the resulting expected coverage as computed by our algorithm. Three representative results for the cases of low, medium, and high call intensity are depicted in Figure 1. The figure shows that the best bound for the expected coverage increases with the number of ambulances in accordance with an S-curve, which is initially convex and then concave. Initially, the curve is close to linear, but ever so slightly convex. After the inflection point, each additional ambulance adds less additional coverage, because there are fewer and fewer uncovered calls and it becomes increasingly difficult to cover these uncovered calls. The behavior of the expected coverage as a function of the number of ambulances is similar to, for example, the admission probability as a function of the number of servers in the Erlang B loss model and the no-delay probability as a function of the number of servers in the Erlang $\mathrm{C}$ delay model $(M / M / c$ queue) with abandonments. We note that the expected coverage in our model will not necessarily approach $100 \%$ as the number of ambulances approaches infinity, because some of the demand locations may be so far from the closest existing station that the probability of coverage will be low no matter how many ambulances are allocated to that station.

\section{Weekly scheduling of ambulances}

We now turn to the main problem of scheduling ambulance crews. We constructed two integer programming models, their main difference lying in the objective function. Additional notation required to state our models follows. 


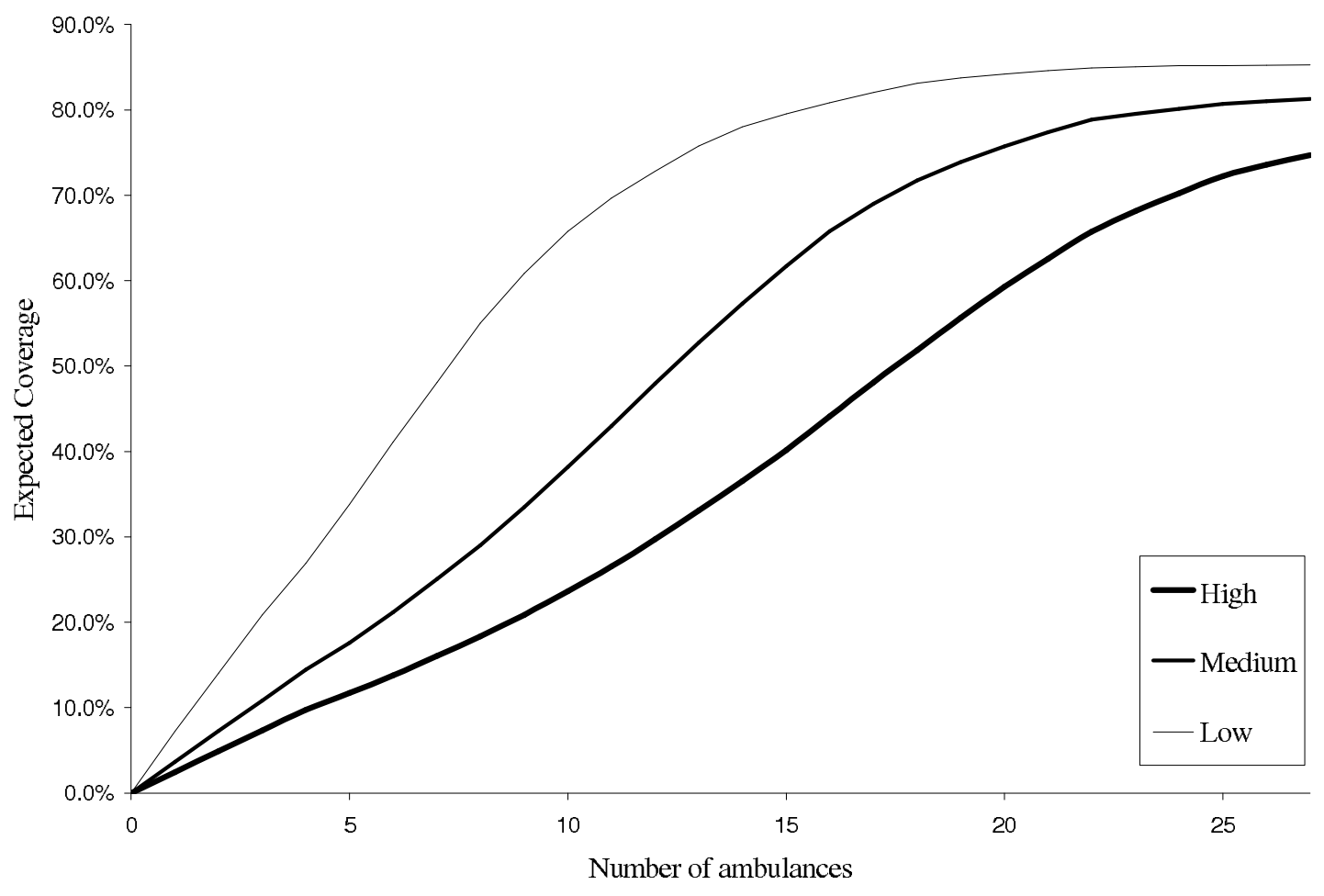

Figure 1: Expected coverage versus number of ambulances

\section{Notation:}

$\delta_{i j}:$ Additional number of expected calls covered at hour $i$ by adding the $j^{\text {th }}$ ambulance. This value is precomputed as the difference of expected coverage for locating $j$ ambulances and $j-1$ ambulances at the $i^{\text {th }}$ hour.

$h_{s} \quad$ : The number of working hours for shift $s$.

$e_{i}$ : The average number of calls received during hour $i$.

$a_{i s}: \quad 1$ if shift pattern $s$ includes hour $i$ and 0 otherwise.

$\alpha$ : The average amount of work hours required to serve a call.

$\tau$ : The number of hours in the planning horizon.

$\sigma$ : The number of shift patterns. 
$\beta$ : The benchmark budget, computed as the total amount of work hours required to serve all calls, i.e., $\beta=\alpha \sum_{i=1}^{\tau} e_{i}$.

$\gamma$ : A parameter denoting the amount of budget allocated in terms of the benchmark budget.

\subsection{Model 1: Maximizing aggregate expected coverage}

As stated in the introduction, our first model aims to maximize the aggregate expected coverage, i.e., the ratio of the sum of the expected number of calls covered during every hour to the total number of calls. Since we consider coverage to be the primary indicator of quality of service, this model aims to maximize the performance of the system. Let $x_{s}$ be equal to the number of ambulance crews scheduled to work on shift $s$, and let $y_{i j}$ be equal to 1 if the total number of ambulance crews during hour $i$ is at least $j, 0$ otherwise. Our first model is then:

(SSP1)

$$
\operatorname{maximize} \sum_{i=1}^{\tau} \sum_{j=1}^{\zeta} \delta_{i j} y_{i j} / \sum_{i=1}^{\tau} e_{i}
$$

subject to

$$
\begin{aligned}
& \sum_{s=1}^{\sigma} a_{i s} x_{s}=\sum_{j=1}^{\zeta} y_{i j} \quad(i \in\{1, \ldots, \tau\}) \\
& y_{i j} \leq y_{i, j-1} \quad(i \in\{1, \ldots, \tau\}, j \in\{2, \ldots, \zeta\}) \\
& \sum_{j=1}^{\tau} y_{i j} \geq\left\lceil\alpha e_{i}\right\rceil \quad(i \in\{1, \ldots, \tau\}) \\
& \sum_{s=1}^{\sigma} h_{s} x_{s} \leq\lfloor\gamma \beta\rfloor \\
& x_{s} \in \mathbb{N} \quad(s \in\{1, \ldots, \sigma\}) \\
& y_{i j} \in\{0,1\} \quad(i \in\{1, \ldots, \tau\}, j \in\{1, \ldots, \zeta\}) .
\end{aligned}
$$


Constraints (6) set the sum of the number of crews scheduled to shifts that are active during a given hour to be equal to the number of ambulances available in that hour. Constraints (7) state that the $j^{\text {th }}$ ambulance can be available only if the $j-1^{\text {st }}$ is available. Constraints (8) set the lower bound on the ambulances available in a given hour as the number of work hours required to serve all calls in that hour. Note that the constraints (7) for a given hour can be discarded if the constraints (8) put the minimum number of ambulances in that hour is above the inflection point of the S-curve. Finally, constraint (9) limits the ambulance crews in terms of maximum work hours that can be afforded. The right hand side of (9) is stated in a parametric way for ease of experimentation.

\subsection{Model 2: Maximin expected coverage, maximum aggre- gate expected coverage}

Although the first model captures the essence of the system at hand, it disregards the concept of equity. In order to cover more calls, it could keep the number of ambulances at the bare minimum at hours with low call intensity and place more ambulances at hours with peak call intensity. A remedy to this problem is to maximize the minimum expected coverage over every hour. However, this approach may result in an underutilization of system resources since this alternative objective function does not differentiate between optimal solutions with differing aggregate expected coverage values. Our aim should then be to find the solution with maximin expected coverage and maximum aggregate expected coverage. Consequently our second model is lexicographically multiobjective, where the first objective is to maximize the minimum expected coverage over every hour, and the second objective is to maximize the aggregate expected coverage. We write maximize $\left(z_{1}, z_{2}\right)$ to denote a lexicographic maximization with $z_{1}$ being the first objective and $z_{2}$ being the second. Let $w$ be equal to the minimum expected coverage over every hour.

$$
\operatorname{maximize}\left(w, \sum_{i=1}^{\tau} \sum_{j=1}^{\zeta} \delta_{i j} y_{i j} / \sum_{i=1}^{\tau} e_{i}\right)
$$

subject to 


$$
w \leq \sum_{j=1}^{\zeta} \delta_{i j} y_{i j} / e_{i} \quad(i \in\{1, \ldots, \tau\})
$$

and (6), (7), (8), (9), (10), and (11).

Solving SSP2 requires solving two integer programming models sequentially, the first of which is simply the model above with the first objective function. Denoting the optimal objective value of the first stage as $w^{*}$, the second stage problem is:

$$
\operatorname{maximize} \sum_{i=1}^{\tau} \sum_{j=1}^{\zeta} \delta_{i j} y_{i j} / \sum_{i=1}^{\tau} e_{i}
$$

subject to

$$
w^{*} \leq \sum_{j=1}^{\zeta} \delta_{i j} y_{i j} / d_{i} \quad(i \in\{1, \ldots, \tau\})
$$

and (6), (7), (18), (9), (10), and (11).

The first stage maximizes the minimum expected coverage, while the second stage maximizes the aggregate expected coverage subject to the constraint that the minimum expected coverage is greater than or equal to the optimal solution value of the first stage.

We use the output of both models to find the number of ambulances available at each hour. We then allocate these ambulances as determined in the preprocessing phase. 


\section{Computational Results}

We used the platform and data described in Section 3 to experiment with the models presented in the previous section. The first part of the experimentation was to run our tabu search algorithm for every hour of the week and every possible number of ambulances in that hour. The computational effort can be reduced by only considering number of ambulances that satisfy constraint (8) . Note that since a new problem is solved for every different hour, response times that depend on the hour can be easily incorporated into this procedure. If the response times are assumed to be the same for every hour, and several hours of the week have the same demand, then one can just do the computations for one of those hours. We have performed a single replication for each instance. This resulted in a total of $7 \times 24 \times \zeta=7 \times 24 \times$ $27=4536$ runs and required 59.4 CPU hours (2.5 days). Although the computing time is large, every instance of the preprocessing stage is independent of each other and does not require licensed software, which allows parallel computation without tedious implementation. On a computing grid consisting of 32 Linux workstations with 64-bit AMD Opteron CPU's, the wall clock time required to complete the preprocessing phase was a little more than two hours.

We emphasize that the models we have presented in the previous section can use the output of every possible solution method for the ambulance location problem. We have used our tabu search algorithm to obtain results that are as realistic as possible. In the case that no more than one CPU can be allocated to the preprocessing stage, one may opt for less sophisticated ambulance location models such as MEXCLP of Daskin (1983) to save computational effort.

The extra piece of information we needed for the second stage was the $a_{i s}$ matrix of shift patterns. We used a matrix with 15 shift patterns that correspond to the shifts that are in current use by a Canadian EMS operator in a mid-size Canadian city. The first shift pattern is a 24-hour shift denoting two crews working shifts of either $12+12$ or $10+14$ hours using the same ambulance. The next nine shifts are 12-hour patterns with start times at the beginning of every hour from $7 \mathrm{AM}$ to 3 PM. The last four shift patterns consist of 10.5-hour shifts, which we approximated as 11-hour shifts, with start times at 6 AM, 7 AM, 10 AM, and 4 PM. Based on the output of the preprocessing stage, we solved both models from the previous section for values of $\gamma \in\{1.5,2,2.5,3,3.5,4\}$. Both models involve about 4500 variables and constraints. Using $\mathrm{C}++$ and the callable library of CPLEX 10.1, the average computing times per instance for SSP1 and SSP2 were 8.99 and 27.14 CPU seconds, respectively. Figure 2 compares the aggregate expected coverage achieved by the 
two models. Both models behave in a similar manner, starting around $40 \%$ and converging to $80 \%$ at $\gamma=4$, at which point the system saturates. The average difference is $0.36 \%$ and the maximum difference is $1.17 \%$ at $\gamma=2$. We conclude that the emphasis on equity does not result in a severe loss in aggregate expected coverage.

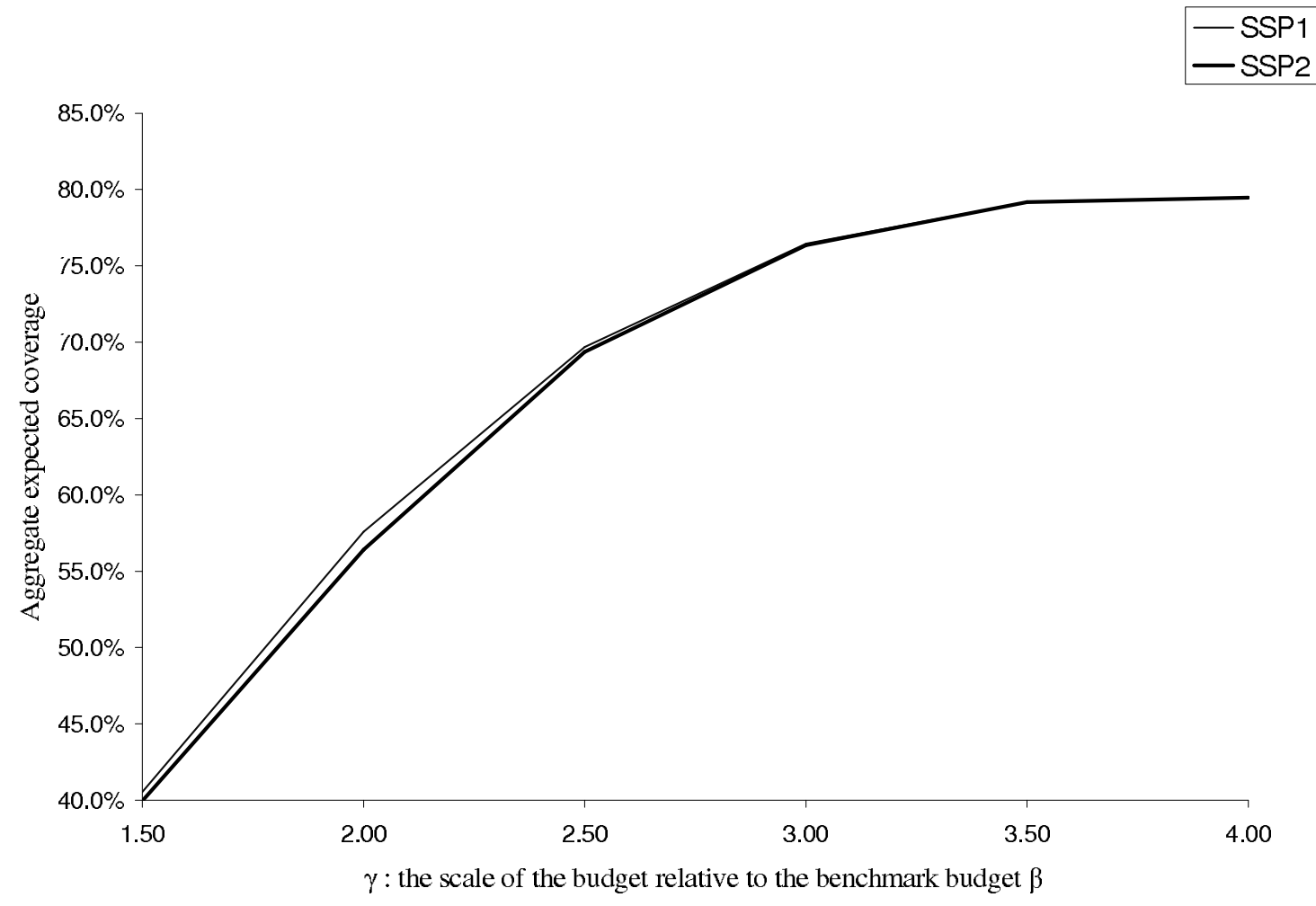

Figure 2: Aggregate expected coverage versus $\gamma$

Figure 3 compares the minimum expected coverage over all hours of the week for the two models. The difference is more pronounced in this case, with an average of $6.19 \%$ and a maximum of $18.34 \%$ for $\gamma=2$. This gives us grounds to claim that SSP1 lacks the sophistication to handle equity while maximizing performance, whereas SSP2 successfully maximizes equity with a marginal deviation from maximum aggregate performance.

We have also analyzed the variation of the number of ambulances with respect to call intensity. Figure 4 depicts the comparison of the number of ambulances allocated 


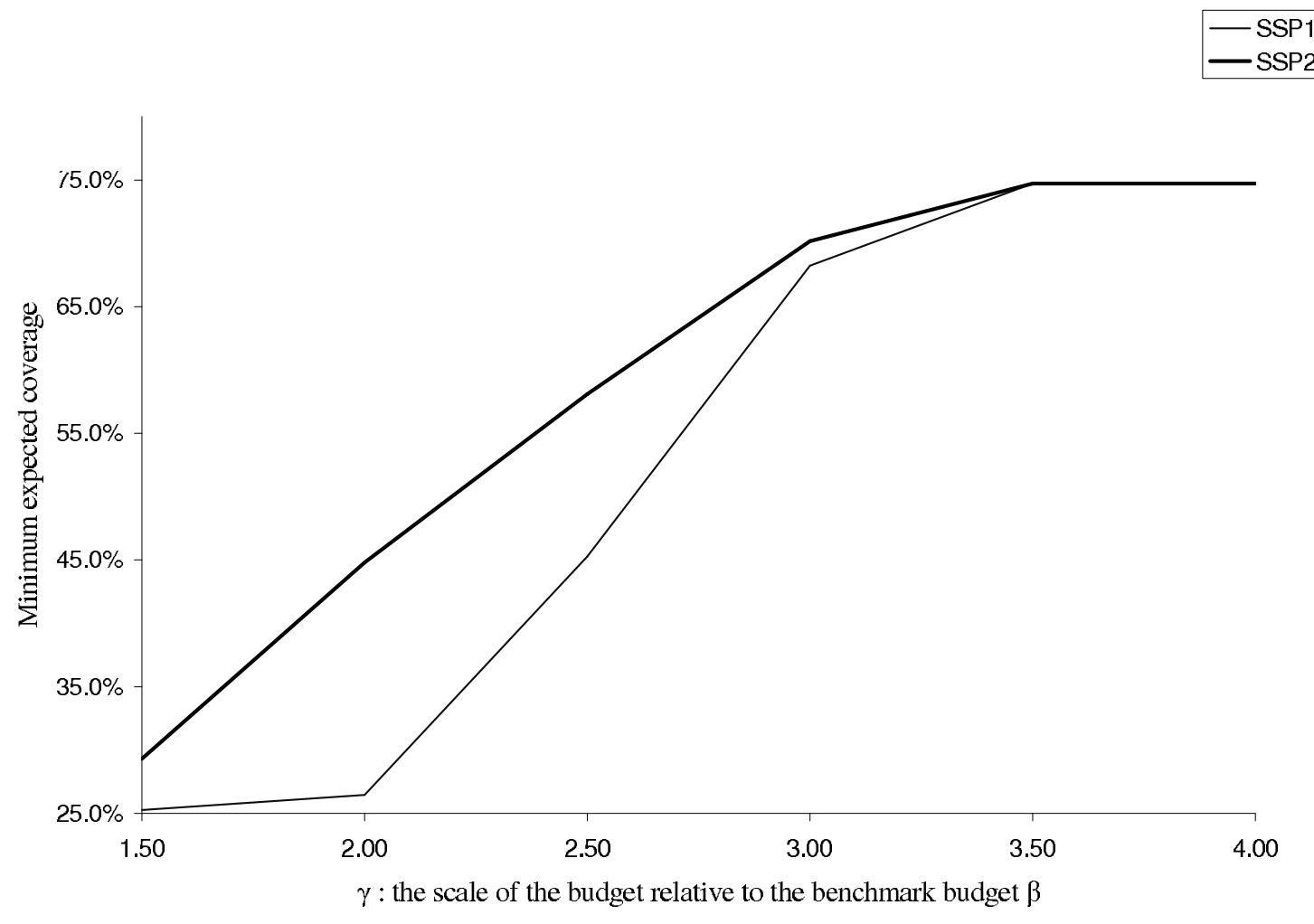

Figure 3: Minimum expected coverage versus $\gamma$

to each hour of the week by both models, and the pattern of the demand intensity. When the budget is low $(\gamma=1.5)$, the staffing curves are driven primarily by the hourly minimum staffing requirements (8) and are therefore similar for both models. When the budget is large $(\gamma=3.5$ or 4$)$, the models behave identically. For more realistic intermediate budgets $(\gamma=2$ or 2.5 , corresponding to ambulance utilization of 40 to $50 \%$ ), the SSP1 model places more emphasis on the peak intensity hours and relatively less emphasis on the low intensity hours. SSP2, on the other hand, is more stable, with a staffing curve that is roughly proportional to the demand intensity. 

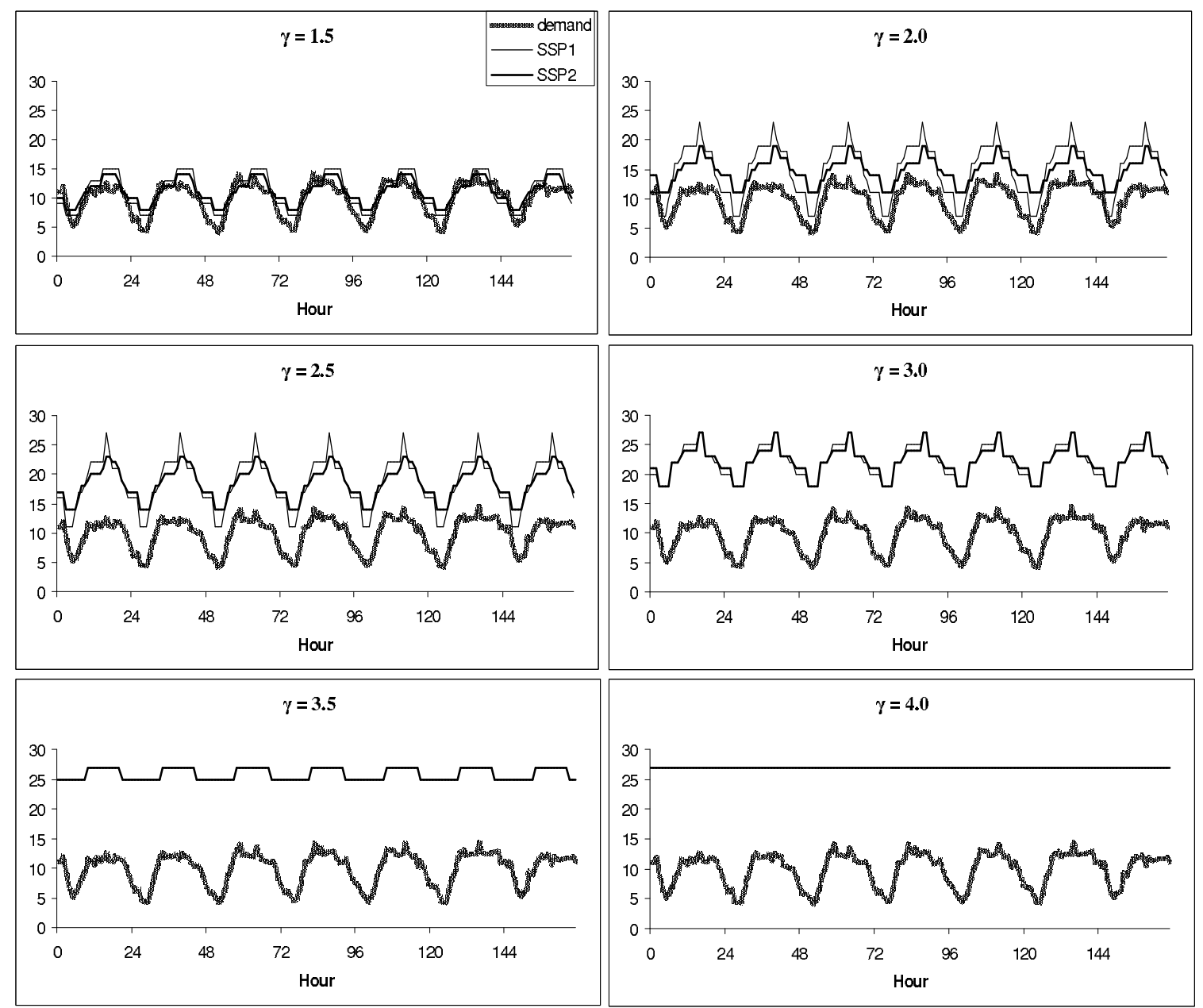

Figure 4: Number of ambulances per hour based on the output of both models. Note that both curves closely follow the demand pattern, with changes in amplitude. 


\section{Conclusions}

In this study we have analyzed the problem of scheduling ambulance crews to shifts in order to maximize coverage. The subproblem of locating the ambulances at stations was solved using a tabu search algorithm, which was empirically shown to outperform the previous approaches in the literature. Two integer programming models were constructed for the problem. Both require the outcome of allocating a given number of ambulances to a given time slot in the planning horizon. The first model emphasizes performance, i.e., maximizing the aggregate expected coverage. The second model is a lexicographic multiobjective model maximizing equity first, i.e., the minimum of hourly expected coverage and the performance second. A computational experiment with real data was conducted. The experiment consists of a parallel preprocessing phase regarding the tabu search algorithm, and running the models on the output of the preprocessing phase. The outputs of the models were graphically analyzed. Our results indicate that the second model can handle the maximization of equity with an average of $0.29 \%$ and a maximum of $1.44 \%$ loss in performance.

Acknowledgments: This work was partially funded by the Canadian Natural Sciences and Engineering Research Council under grants 39682-05 and 203534-07. This support is gratefully acknowledged. We thank the Edmonton and Calgary EMS departments for providing access to data, and Dan Haight and Matt Stanton for their assistance with data preperation.

\section{References}

L. Brotcorne, G. Laporte, and F. Semet. Ambulance location and relocation models. European Journal of Operational Research, 147:451-463, 2003.

S. Budge, A. Ingolfsson, and E. Erkut. Approximating vehicle dispatch probabilities for emergency service systems with location-specific service times and multiple units per location. Operations Research, forthcoming, 2008.

M.S. Daskin. A maximum expected covering location model: formulation, properties, and heuristic solution. Transportation Science, 17:48-70, 1983.

M.S. Daskin. Location, dispatching, and routing model for emergency services with stochastic travel times. In A. Ghosh and G. Rushton, editors, Spatial Analysis and Location Allocation Models. Van Nostrand Reinhold, New York, 1987. 
E. Erkut, A. Ingolfsson, T. Sim, and G. Erdoğan. Computational comparison of five maximal covering models for locating ambulances. Geographical Analysis, forthcoming, 2008.

A.T. Ernst, H. Jiang, M. Krishnamoorthy, and D. Sier. Staff scheduling and rostering: A review of applications, methods and models. European Journal of Operational Research, 153:3-27, 2004.

J.B. Goldberg. Operations research models for the deployment of emergency services vehicles. EMS Management Journal, 1:20-39, 2004.

J.B. Goldberg and L. Paz. Locating emergency vehicle bases when service time depends on call location. Transportation Science, 25:264-280, 1991.

L. V. Green, P. J. Kolesar, and J. Soares. Improving the SIPP approach for staffing service systems that have cyclic demand. Operations Research, 49:549-564, 2001.

A. Ingolfsson, S. Budge, and E. Erkut. Optimal ambulance location with random delays and travel times. Health Care Management Science, forthcoming, 2008.

H. Jia, F. Ordonez, and M. Dessouky. A modeling framework for facility location of medical service for large-scale emergencies. IIE Transactions, 39:41-55, 2007.

G. Koole and E. van der Sluis. Optimal shift scheduling with a global service level constraint. IIE Transactions, 35:1049-1055, 2003.

R.C. Larson. Approximating the performance of urban emergency service systems. Operations Research, 23:845-868, 1975.

V. Marianov and C.S. ReVelle. Siting emergency services. In Z. Drezner, editor, Facility Location: A Survey of Applications and Methods, pages 199-222. Springer-Verlag, New York, 1995.

C.S. ReVelle and K. Hogan. The maximum availability location problem. Transportation Science, 23:192-200, 1989.

C. Saydam and M. McKnew. A separable programming approach to expected coverage: an application to ambulance location. Decision Sciences, 16:381-398, 1985.

A.J. Swersey. The deployment of police, fire, and emergency medical units. In A. Barnett, S.M. Pollock, and M.H. Rothkopf, editors, Handbooks in Operations Research and Management Science, Vol. 6: Operations Research and the Public Sector, pages 151200. North Holland, Amsterdam, 1994.

G.M. Thompson. Labor staffing and scheduling models for controlling service levels. Naval Research Logistics, 44:719-740, 1997. 